\title{
Bilateral hyperplasia of the mandibular coronoid processes associated with the nevoid basal cell carcinoma syndrome in an Italian boy
}

\author{
R. Leonardi, ' G. Sorge, ${ }^{2}$ and M. Caltabiano, ${ }^{3}$
}

In this report we present a subject affected by nevoid basal cell carcinoma syndrome (NBCCS), showing also bilateral mandibular coronoid processes hyperplasia, a hitherto unreported association. Our observation of bilateral hyperplasia of the mandibular coronoid processes in a boy with NBCCS may prompt a retrospective and prospective review of other patients affected by this syndrome in order to establish if this anomaly is part of it.
The nevoid basal cell carcinoma syn1 drome (NBCCS) was delineated by Gorlin and Goltz in 1960 and often bears the former's name. ${ }^{1}$ Its prevalence has been estimated at $1 / 56,000$. NBCCS is an autosomical dominant multisystem disorder, although some cases, which represent new mutations, have been described.

The syndrome is characterized by multi-

In brief

- The nevoid basal cell carcinoma syndrome (NBCCS) is a multi-system disorder.

- This syndrome is characterized by multiple basal cell carcinoma, odontogenic keratocysts and congenital skeletal anomalies.

- It is presented as a subject affected by NBCCS showing also bilateral mandibular coronoid hyperplasia.

- Mandibular coronoid hyperplasia is an unreported association.

${ }^{1,3}$ Department of Orthodontics, Istituto di II Clinica Odontoiatrica, Policlinico, Catania University, viale A. Doria 6, Catania, Italy; ${ }^{2}$ Department of Pediatrics, Policlinico, Catania University, viale A. Doria 6, Catania, Italy

Correspondence to: Rosalia Leonardi, Istituto di II Clinica Odontoiatrica, Policlinico, Università di

Catania, Viale A. Doria n.6, Catania, Italy e-mail: rleonard@mbox.unict.it

REFEREED PAPER

Received 05.06.00; Accepted 19.09.00

(C) British Dental Journal 2001; 190: 349-350 ple basal cell carcinomas (BCCs), odontogenic keratocysts of the jaw, congenital skeletal anomalies, ectopic calcification particularly of the falx cerebri, and palm and/or sole pits. Other features include bridging of the sella turcica, mild mandibular prognathism, lateral displacement of the inner canthi, frontal and biparietal bossing, kyphoscoliosis, bifid, missing, fused and/or splayed ribs, imperfect segmentation of cervical vertebrae, ovarian fibromata and lymphomesenteric cysts which tend to calcify, and a short fourth metacarpal. ${ }^{2}$ The clinical expression of the syndrome varies among individuals within the same family and even more so among families.

In this report we present a subject affected by NBCCS, showing also bilateral mandibular coronoid processes hyperplasia, a hitherto unreported association.

\section{Case report}

A 13-year-old boy, with a history of NBCCS, was admitted to the Department of Oral Surgery of Catania University for enucleation of a cyst in the maxilla. The subject was the first child of non-consanguineous parents. He had a sister with no congenital abnormalities. The clinical sign leading to hospitalisation was an asymptomatic tumescence on the left maxillary gingiva. That was associated with displacement and loosening of the left deciduous incisors and canine. Radiographs revealed a large radiolucent defect. The patient underwent a conservative surgical treatment of this cystic lesion. At age 14 the patient was referred to the Department of Orthodontics of the same University for the treatment of his malocclusion.

On clinical orthodontic examination the patient showed limited mandibular excursions. Maximal effort produced a $25 \mathrm{~mm}$ vertical interincisal opening, which could not be increased with the use of tongue

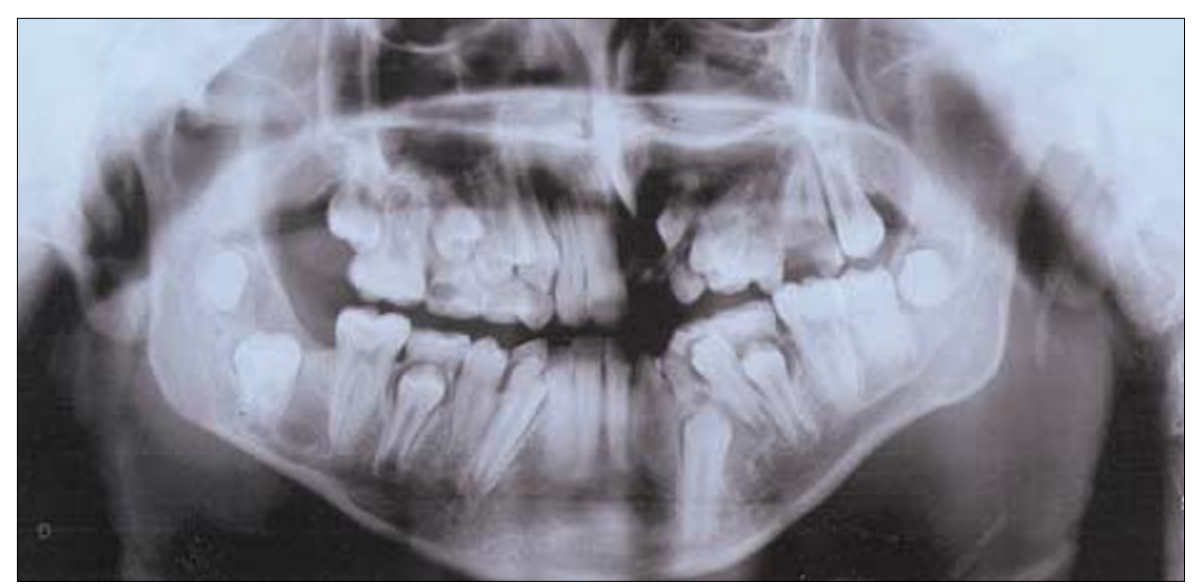

Fig.I The panoramic radiograph, taken I year after the enucleation of the cyst in the maxilla, reveals grossly hyperplastic coronoid processes. Note also the round radiolucencies in the mandible 


\section{case study}

blades or by asking the patient to open his mouth with the mandible in a protruding position. A forced mandibular opening resulted in a dull bilateral pain over both infratemporal regions. Protrusion and lateral excursions were equally limited. There was no muscle or temporomandibular joint pain, deviation on opening or facial asymmetry. A panograph (Fig. 1) and various radiographic views of the mandible were taken and each substantiated the clinical observation of bilateral enlongated coronoid processes. The lateral cephalometric projection (Fig. 2) confirmed the presence of bilateral hyperplasia of the mandibular coronoid processes and also disclosed a bridged sella turcica and an abnormal first cervical vertebra. The radiographic examination of the right hand suggested slight shortening of the fourth metacarpals.

\section{Discussion}

The stigmata originally attributed to NBCCS were multiple basal cell nevi, cysts of the jaws and skeletal anomalies, the latter being a consistent feature of the syndrome. ${ }^{2}$ On the other hand the occurrence of bilateral hyperplasia of the mandibular coronoid processes in NBCCS has not yet been previously described.

Bilateral coronoid hyperplasia is characterized by a progressive limitation in mandibular movement, secondary to mechanical impingement of the enlongated coronoid processes on the posterior surface of the zygomas. ${ }^{3}$ There is usually no facial asymmetry or pain and onset occurs at puberty. About 80 cases of mandibular coronoid hyperplasia have been reported so far in the literature with a marked male preponderance. The etiology of coronoid hyperplasia remains uncertain, with various proposed

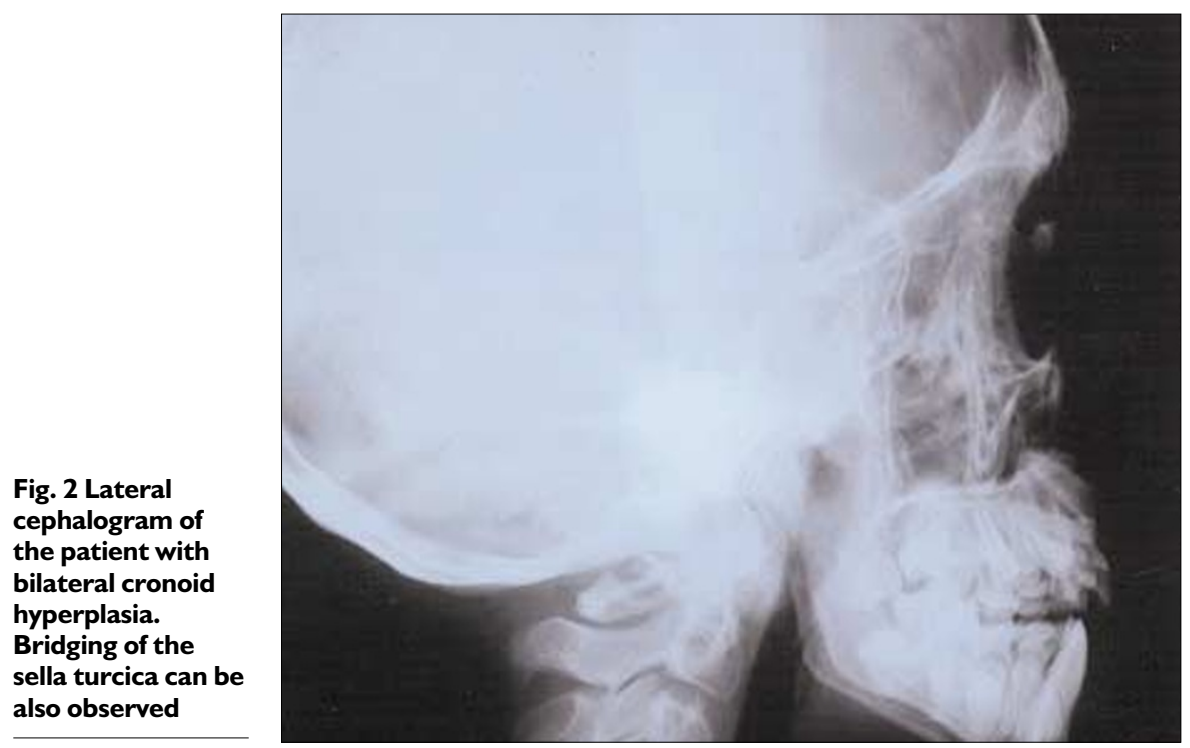

theories. ${ }^{3}$ An endocrine stimulus, increased temporalis activity, trauma, genetic inheritance and familial occurrence have all been suggested, none of which explains the condition. A syndromic relationship has also been described in trismus-pseudocamptodactyly syndrome. ${ }^{4}$

Bilateral coronoid hyperplasia which was detected in this patient was not associated with any family history and seems to be a new skeletal abnormality until now not described in patients affected by NBCCS syndrome.

\section{Conclusion}

Mandibular hypomobility usually draws the clinician's attention to the temporomandibular joints and masticatory muscles. Although abnormalities of the coronoid process are considered to be less common they should not be ignored in the differential diagnosis. History of the patient's complaint, clinical examination of the TMJ and coronoid processes imaging are important factors in formulating the diagnosis.

Our observation of bilateral hyperplasia of the mandibular coronoid processes in a boy with NBCCS may prompt a retrospective and prospective review of other patients affected by this syndrome in order to establish if this anomaly is part of it.

1 Gorlin R J. From oral pathology to craniofacial genetics. Am J Med Genet 1993; 46: 317-334.

2 Shanley S, Ratcliffe J, Hockey A, Haan E, Oley C, Ravine D, Martin N, Wicking C, ChenevixTrench G. Nevoid basal cell carcinoma syndrome: review of 118 affected individuals. Am J Med Genet 1994; 50: 282-290.

3 Totsuka Y, Fukuda H. Bilateral coronoid hyperplasia. Report of two cases and review of the literature. JCraniomaxillofac Surg 1991; 19: 172-177.

4 Yamashita D D R, Arnet G F. Trismus pseudocamptodactyly syndrome. J Oral Surg 1979; 37: 744-746. 